E. Nettis ${ }^{1}$, F. Bonifazi ${ }^{2}$, S. Bonini ${ }^{3}$, E. Di Leo ${ }^{1,4}$, E. MagGi ${ }^{5}$, G. Melioli ${ }^{6}$, G. Passalacqua ${ }^{7}$, G. Senna ${ }^{8}$, M. Triggiani ${ }^{9}$, A. Vacca ${ }^{1}$, G.W. CanOnica $^{7}$

\title{
Molecular diagnosis and the Italian Board for ISAC
}

\author{
${ }^{1}$ Section of Allergy and Clinical Immunology, Dept. of Internal Medicine, University of Bari, Bari, Italy \\ ${ }^{2}$ Allergy Unit, Dept. of Respiratory and Allergic Diseases, Azienda Ospedaliera Umberto I, Torrette, Ancona, Italy \\ ${ }^{3}$ Institute of Translational Pharmacology, Italian National Research Council, Second University of Naples, Naples, Italy \\ delle Fonti, Bari, Italy \\ ${ }^{5}$ Section of Immunoallergology, University of Florence, Florence, Italy \\ ${ }^{6}$ Laboratories of Analysis, Istituto Giannina Gaslini, Genoa, Italy \\ ${ }^{7}$ Allergy and Respiratory Diseases, IRCCS IST San Martino, University of Genoa, Genoa, Italy \\ ${ }^{8}$ Allergy Unit, Azienda Ospedale Università, Verona, Italy \\ ${ }^{9}$ Immunoallergology Unit, University of Salerno, Salerno, Italy
} ${ }^{4}$ Section of Allergy and Clinical Immunology, Internal Medicine Unit, Ente Ecclesiastico Ospedale "F. Miulli", Acquaviva

\section{KEY WORDS \\ Component Resolved Diagnostic; Immuno Solid-phase Allergen Chip (ISAC); Italian Board for ISAC}

\section{Corresponding author}

Elisabetta Di Leo

Section of Allergy and Clinical Immunology Dept. of Internal Medicine, University of Bari Piazza G. Cesare 11, 70124 Bari, Italy

Phone: +390805593605

E-mail: elisabettadl@alice.it

\begin{abstract}
Summary
The Component Resolved Diagnostic (CRD) approach has been developed when highly purified or recombinant allergen molecules have become available. These molecules are the allergenic proteins toward which the specific and clinically relevant IgE immune response is directed. So, the identification of protein families and cross-reactivity patterns of importance in allergy have been possible. The Italian advisory BOARD for ISAC was born: to evaluate the advantages, disadvantages and placement in diagnosis of CRD studying its application in allergic patients; to facilitate the interpretation of molecular diagnostics for clinical allergists; to evaluate the effectiveness of CRD in improving diagnostic risk assessment and early preventive treatment of allergic diseases. In the last years, its fields of interest have been: the evaluation of the performance of CRD on multi-sensitized allergic patients with respiratory symptoms and on poly-sensitized athletes; the evolution of IgE repertoire directed to single allergenic components by evaluating allergic patients with different age at a molecular level; the relevance of results obtained using allergen microarray technique for describing the IgE repertoire in allergic patients by reviewing the main articles focused on CRD published in the last 2 years; the need for an educational program focused on this new diagnostic tool also through the creation of an exhaustive and interactive explanation of the laboratory report molecular allergy; the investigation of the performance and potential additional diagnostic values of the ISAC microarray in a real-life clinical setting, taking into account also the economic values.
\end{abstract}

\section{Introduction}

Many different allergens share common protein epitopes, and the same specific $\operatorname{IgE}(\mathrm{s} \operatorname{IgE})$ antibody can bind to proteins with similar structures present in different allergen sources, thus recognizing homologous allergens from different sources. Skin Prick Test (SPT) or allergen-sIgE tests cannot resolve this question (1). Also, a common assumption in SPT allergy diagnostic practice is that only a limited number of allergens are routinely as- sayed using both SPT and sIgE detection: a large number of SPT may be disagreeable for the patient and a large number of sIgE tests can be both expensive and blood sample volume demanding. Furthermore, many patients with allergic symptoms are multi-sensitized, and it is often difficult to assess whether this multi-sensitization is due to a genuine co-sensitization or to cross-reactivity (1).

The Component Resolved Diagnostic (CRD) approach (2) has been developed when highly purified or recombinant allergen 
molecules have become available (1). These molecules are the allergenic proteins toward which the specific and clinically relevant IgE immune response is directed. This development has enabled the identification of protein families and cross-reactivity patterns of importance in allergy $(1,3)$.

So, the protein microarray technology and specifically the allergen microarray, allow simultaneous analysis and monitoring of patient-specific antibody profiles for a previously unknown variety of allergens in a single analytical step (4).

Immuno Solid-phase Allergen Chip (ISAC), ImmunoCAP ISAC®, (Thermo Fisher Scientific, Uppsala, Sweden) is the first in vitro diagnostic tool based on modern biochip technology: it is a miniaturized immunoassay platform, that allows multiplex measurement of specific IgE antibodies for many (actually 112) natural purified and recombinant allergen molecules using only $30 \mu \mathrm{l}$ of serum or plasma (5). The method provides components spotted on a solid support (biochip). In a two-step assay, IgE antibodies from the patient's serum bind to the allergen components. After a short washing step, allergen-bound IgE antibodies are detected by a fluorescence-labeled anti-IgE antibody. Test results are measured with a biochip scanner and evaluated using a dedicated software. ImmunoCAP ISAC® is a semiquantitative test and results are reported in ISAC Standardized Units (ISU) (5).

\section{General consideration on CRD}

Extractive allergen preparations (used for the skin prick test, for specific $\operatorname{IgE}$ detection and classic immunotherapy) are a mixture of several different molecules containing non allergenic proteins, specific allergens (i.e., molecules that can be directly associated with that allergen), pan-allergens (molecules that are present not only in that mixture, but also in other mixtures of similar sources and constituted by highly homologous molecules) and, finally, cross-reacting allergens (i.e., molecules that are present in one allergen, but which also may be present in other not strictly related allergens, because of a certain degree of homology at least in the primary structure of the protein) (6). Conventionally, these molecules are defined as components, and the mixture of different components constitutes the allergen. A specific component is in general responsible for a genuine sensitization to that allergen, whereas a sensitization to pan-allergens or cross-reacting allergens cannot be considered genuine. Components are available for in vitro diagnosis in two different forms: as recombinant allergens (i.e., as allergens cloned in prokaryotic or eukaryotic cells using genetic engineering techniques) and as highly purified extractive molecules (6). Components are named using a conventional notation. Consequently, the first 3 letters (such as Phl, Bet, and so forth) correspond to the first 3 letters of the Linnaean name of the source (in this case Phleum, Betula). The fourth letter (in lower case, as dictated by living organism nomenclature) indicates the first letter of the "second" name of the source. Thus, a molecular component of Phleum pratense is defined as Phl p. Finally, a number is added to the letter, distinguishing each component from the others. Phl p 1 indicates that the first component identified (and in general cloned) is Phleum pratense. Finally, the letter " $r$ " or " $n$ " preceding the component name is indicative of the origin (namely, "recombinant" or "natural"). In general, an $\mathrm{IgE}$ repertoire directed to a specific component is suggestive of genuine sensitization. Any other IgE specificity cannot be considered genuine unless the clinical indication is so typical as to strongly associate that allergen to the patient's history $(6,7)$.

In the "molecular allergy era", monosensitization seems to be extremely difficult to identify since the patient is in general sensitized to more than 1 component of the same allergen source. Thus, the sensitization to a pan-allergen is very frequently associated with a specific sensitization. In the presence of $\operatorname{IgE}$ directed to a large number of components belonging to the same family (such as PR-10, the Bet v1 homolog), the original sensitization should be defined. In this context, a gradient of capacities of sensitizing patients is frequently observed in poly-sensitized subjects and, in a significant number of cases, the sensitizing source can be identified as the component with the highest score (6).

\section{Italian Board for ISAC (IBI)}

\section{IBI objectives}

The Italian advisory BOARD for ISAC was formed in 2009 with the following objectives:

- to evaluate the advantages, disadvantages and placement in diagnosis of CRD studying its application in allergic patients (to evaluate if using CRD for allergic patients could add important information not directly available using standard SPT and $\operatorname{sgE}$, and if this method may play a key role in improving clinical management of poly-sensitized allergic patients, etc.);

- to facilitate the interpretation of molecular diagnostics for clinical allergists through the publication of reports and reviews, but also by introducing the new molecular method at congresses and symposia;

- to evaluate the effectiveness of CRD in improving diagnostic risk assessment and early preventive treatment of allergic diseases.

\section{IBI fields of interest}

Since the Board was established in 2009, its fields of interest (table 1), concerning the CRD, have been the evaluation of:

- the performance of CRD on multi-sensitized allergic patients with respiratory symptoms in a cross-sectional observational Italian study (1); 
- the study of the sensitization pattern in a population of poly-sensitized patients with respiratory allergy living in a restricted geographical area in the Northwest Italy (8);

- the evolution of $\operatorname{IgE}$ repertoire directed to single allergenic components by evaluating allergic patients belonging to 6 groups stratified according to age at a molecular level (9);

- the relevance of results obtained using allergen microarray technique for describing the $\operatorname{IgE}$ repertoire in allergic patients, by reviewing the main articles focused on CRD published in the last 2 years (10);

- the need for an educational program focused on this new diagnostic tool also through the creation of an exhaustive and interactive explanation of the laboratory report molecular allergy (6);

- the potential diagnostic added value of the microarray technology detecting IgE antibodies to specific or cross-reacting allergen components in poly-sensitized athletes (11);

- the investigation of the performance and potential additional diagnostic values of the ISAC microarray in a real-life clinical setting, taking into account also the economic values (12).

\section{Results of the studies of IBI}

A total number of 321 sera from multi-sensitized adult Italian patients with well defined respiratory allergy and 92 control subjects with no history of allergy-like respiratory symptoms, were studied using both conventional extract based allergen-sIgE and a CRD diagnostic microarray tool in a prospective cross-sectional multicenter study (May 2009 - February 2010). The study involved 5 sites distributed all over Italy: Ancona, Bari, Cuneo, Florence and Genoa. In the context of this clinical study the primary objectives were to evaluate the technical performance of the ISAC (containing 103 different allergen components/molecules originating from 47 allergen sources) based CRD tool, the relation of ISAC results with extract based sIgE results with ImmunoCAP Allergens in the Phadia 250 system and finally the ability to detect and resolve patterns of true co-sensitization and cross-reactivity.

The results obtained clearly demonstrated that ImmunoCAP ISAC was an effective tool for allergen sIgE measurements and that the technical performance of ISAC and the correlation with "classic" sIgE assays was excellent. Important information on co-sensitization to other allergens than the expected aeroallergens was obtained. In addition, sensitization to cross-reacting components explaining some of the perceived multisensitization was detected. Elevated IgE antibodies to Profilins, CCD (Ana c 2) and calcium-binding proteins were detected, probably due to sensitization to grasses and trees in this study. The possibility of identifying the presence of $\operatorname{IgE}$ antibodies to Profilins in pa-

Table 1 - Italian Board for ISAC scientific production

\begin{tabular}{|l|c|c|c|}
\hline Title & Authors, Year & Study design & Journal \\
\hline $\begin{array}{l}\text { The ImmunoCAP ISAC molecular allergolo- } \\
\text { gy approach in adult multi-sensitized Italian } \\
\text { patients with respiratory symptoms. }\end{array}$ & Melioli G et al., 2011 & $\begin{array}{c}\text { Cross-sectional } \\
\text { observational study }\end{array}$ & Clinical Biochemistry \\
\hline $\begin{array}{l}\text { Sensitization profiles in poly-sensitized patients } \\
\text { from a restricted geographical area: further } \\
\text { lessons from multiplexed component resolved } \\
\text { diagnosis. }\end{array}$ & Rossi RE et al., 2011 & Retrospective study & $\begin{array}{c}\text { European Annals of } \\
\text { Allergy and Clinical } \\
\text { Immunology }\end{array}$ \\
\hline $\begin{array}{l}\text { The IgE repertoire in children and adolescents } \\
\text { resolved at component level: a cross-sectional } \\
\text { study. }\end{array}$ & Melioli G et al., 2012 & $\begin{array}{c}\text { Cross-sectional ob- } \\
\text { servational study }\end{array}$ & $\begin{array}{c}\text { Pediatric Allergy and } \\
\text { Immunology }\end{array}$ \\
\hline $\begin{array}{l}\text { The added value of allergen microarray tech- } \\
\text { nique to the management of poly-sensitized } \\
\text { allergic patients. }\end{array}$ & Melioli G et al., 2012 & Review & $\begin{array}{c}\text { Current Opinion on } \\
\text { Allergy and Clinical } \\
\text { Immunology }\end{array}$ \\
\hline $\begin{array}{l}\text { Molecular allergy diagnosis: we need to become } \\
\text { more knowledgeable. }\end{array}$ & Melioli G et al., 2012 & Guest editorial & $\begin{array}{c}\text { Annals of Allergy and } \\
\text { Asthma Immunology }\end{array}$ \\
\hline $\begin{array}{l}\text { Microarray evaluation of specific IgE to allergen } \\
\text { components in elite athletes. }\end{array}$ & Bonini M et al., 2012 & $\begin{array}{c}\text { Cross-sectional } \\
\text { observational study }\end{array}$ & Allergy \\
\hline $\begin{array}{l}\text { The additional values of microarray allergen } \\
\text { assay in the management of poly-sensitized } \\
\text { patients with respiratory allergy. }\end{array}$ & Passalacqua G et al., 2012 & Multicentric study & Allergy 2013 in press \\
\hline
\end{tabular}


tients with plant allergy is an interesting opportunity enabled by CRD, which may explain both numerous positive extract based tests, in vivo as well as in vitro, and Oral Allergy Symptoms to fruits and vegetables in the absence of $\operatorname{IgE}$ antibodies to PR10 proteins. Along this line, the possibility of excluding the presence of an allergy to profilins in patients with plant allergy, is an interesting added value of this technique for the evident therapeutic outcomes that it implies. Elevated $\operatorname{IgE}$ antibodies to Lipid Transfer Proteins were detected in 55\% of the patients and Storage Proteins (seed) in 8\%; these sensitizations imply an increased risk of severe systemic reactions. ImmunoCAP ISAC can offer a wider representation of the $\operatorname{IgE}$ repertoire of the patients, with evident advantages in patients with suspected multi-sensitization to panallergens and/or cross-reacting allergens (1). In the light of these findings, ImmunoCAP ISAC is also particularly powerful in poly-sensitized patients not only to detect the actual molecular component involved in the allergy, but also to rule out cross reacting allergens and other components (such as LTP) that can be responsible of adverse events (1).

In a subsequent retrospective study, the sensitization profile by means of microarray dosing specific IgE towards 103 allergenic components in a population of poly-sensitized patients with respiratory allergy symptoms living in a restrict geographical area (Cuneo, Northwest Italy) was analyzed. The results showed that in this geographical area, Phl p 1 allergen was the most common sensitization, followed by Phl p 5 reflecting clearly the local flora, since in this area grasses are largely present. An interesting finding of this study is that the stronger allergens, capable of stimulating high levels of specific $\operatorname{IgE}$, are in descending order Phl p 1, Phl p 5, Der f 2, Der p 2, Bet v 1, Der f 1, Der p 1, Ole e 1 and Fel d 1. This data should be taken into account by allergen extract producers in order to provide clinicians with improved products for diagnosis and immunotherapy (8).

It is well known that allergy evolves at clinical level from the birth to adulthood, and this has been clearly demonstrated also at a level of sensitization profile. Since little information is available on the evolution of the $\operatorname{IgE}$ repertoire directed to single allergenic components, the Authors, in a cross-sectional, observational study, analyzed the evolution of the $\mathrm{IgE}$ repertoire at component level by evaluating serum samples from 901 allergic patients (both respiratory and food allergy), stratified in 6 groups according to age (from early infancy to adult age) by means of ImmunoCAP ISAC (for the identification of specific IgE towards 103 different allergen components) (9). The main finding was a clear time-related modification of the $\operatorname{IgE}$ repertoire at component level. Along this line, the percentage of positive components (representative of the $\operatorname{IgE}$ repertoire) during the first period of life was relatively small $(20-30 \%$ of the 103 ISAC molecules), and this percentage increased during the subsequent periods. As expected, food-related components (in particular those of milk and egg) were the most frequently recognized in the earliest ages, whereas specific IgE to plant allergens appeared invariably later. Nonetheless, IgE specific to mite components was the most represented in all age classes. Of note, specific IgE against cross-reacting allergens was virtually absent in the first years and tended to appear only after the age of 6. The modification of $\mathrm{IgE}$ repertoire at component level, from birth to adolescence, seems consistent with the clinical characteristics of the allergic march. This fact is sustained by the initial mono- or oligo-sensitization, followed by poly-sensitization during adolescence, the increase in IgE positive components over time, early sensitization to milk and egg components characterized by a low number of specific IgE followed, after the third year of life, by sensitization to inhalant allergens belonging to the mite family and characterized by a high concentration of specific IgE. In addition, cross-reacting components and pan-allergens were negative up to 6-10 yrs, becoming positive later on, in terms of number and ISU score of components identified by specific IgE (9).

The ISAC Board also focused its attention on a particular population, characterized by elite athletes that, as have been reported, have a very high and increasing prevalence of allergic sensitization and diseases. Furthermore over $80 \%$ of allergic athletes are poly-sensitized. Seventy-two poly-sensitized athletes according to SPT with different allergic phenotypes (asthma, rhino-conjunctivitis; food allergy and/or oral allergy syndrome; no clinical symptoms) and two different control populations (poly-sensitized sedentary subjects with respiratory allergy and 20 healthy athletes with negative SPT) were studied for detecting specific $\mathrm{IgE}$ both to allergen extracts (ImmunoCAPsIgE) and to allergen components (ImmunoCAP ISAC), in order to evaluate the potential diagnostic added value of ImmunoCAP ISAC in detecting $\operatorname{IgE}$ antibodies to specific or cross-reacting allergen components (11). ImmunoCAP ISAC detected the presence of sIgE in $90 \%$ of poly-sensitized athletes and in $100 \%$ of allergic controls. The pattern of positivity towards the 103 components tested differed from a subject to another, even in those with the same sensitization to allergen extract SPT or sIgE. Based on the ISAC results, poly-sensitized athletes were classified into the following prototypical patterns, represented separately in the clinical phenotypes studied: a) One single predominant specific allergen positivity; b) sIgE to two or more non-cross-reacting allergens; c) sIgE to cross-reacting allergens; and d) sIgE to components potentially responsible for severe allergic reactions. On the basis of these results, the ImmunoCAP ISAC may represent a valuable complement for diagnosis and management of poly-sensitized athletes (11).

The IBI, also performed a large population-based (318 allergic patients and 91 controls) study involving six Italian allergy units (Ancona, Bari, Cuneo, Florence, Genoa and Rome) to inves- 
tigate the performance and the potential additional diagnostic values of the ISAC microarray in poly-sensitized subjects in a real-life clinical setting, taking into account also the economic values. In this multicentre study, allergists were required to carefully record diagnosis and treatment of consecutive patients referred for asthma/rhinitis, using the standard methodology (history, SPT, IgE assay). Then, a microarray allergen assay was carried out. Clinicians were required to review their diagnosis/ treatment according to microarray results. An economical analysis was also performed. The clinicians reported at least one additional information from the microarray in about $60 \%$ of patients, this resulting in therapeutic adjustments. In $66 \%$ of patients IgE to pan-allergens were detectable, being this clinically relevant in $38 \%$ of patients with poly-sensitization to pollens. The microarray assay proved to be economically advantageous, when more than 10 recombinant/purified molecules would otherwise have been required for a satisfactory diagnosis (12).

\section{Conclusion}

Diagnostics using CRD offer possibilities not available with extract based standard techniques such as SPT and allergen sIgE tests, in fact, CRD effectively helps in distinguishing between specificity and cross-reactivity in patients with suspected multi-sensitization to various allergens (1). This may have a significant impact on the patient management in terms of risk assessment, advice to avoid allergens, patient selection for immunotherapy, and immunotherapy regime (13).

ImmunoCAP ISAC is also particularly powerful in poly-sensitized patients (in which an accurate aetiological diagnosis is complex) not only to detect the actual molecular component involved in the allergy, but also to rule out cross reacting allergens and other components (such as LTP) that can be responsible for adverse events (1).

However, the current version of ImmunoCAP ISAC cannot fully substitute the use of SPT or allergen sIgE tests, for its apparent inability to detect very low concentrations of $\operatorname{IgE}$ antibodies. Moreover, the panel of 103 allergens does not include all known allergens, and some clinically relevant ones are still missing: for example Ambrosia and Parietaria are represented only by a single component (Amb a 1 and Par j 2, respectively) (9). Therefore, at present, a combined strategy of using SPT or allergen sIgE (complete allergens) together with CRD tools (purified or recombinant allergen components) seems to offer the most complete approach (1). Most likely the addition of missing components, will improve the performance of ISAC (9).

The fact that allergen microarray technology had offered a very large amount of information in the last years is certain. Not only is a more complete description of the $\operatorname{IgE}$ repertoire in different patients, ages and diseases now available, but also the impact of this novel approach to the solution of complex problems of allergy sensitization, even in terms of system biology approach, has been proposed. Nevertheless, from the allergist point of view, the position of allergen microarrays in the flowchart of allergy diagnosis should be better defined. Indeed, despite the indication that allergen microarray, thanks to its characteristics, could be used as first-line assay, other evidence, including costs and diffusion of the technology, indicates that its position is behind extract-based SPT and sIgE, in an accurately selected population of poly-sensitized patients, particularly those with a combined inhalant and food allergy (9). In particular, in poly-sensitized patients where an accurate aetiological diagnosis is complex, due to the presence of sensitizations to both genuine allergens and cross-reacting components.

Results obtained by IBI studies suggest that, at least at component level, a radical change in the IgE repertoire occurs starting from the age of 6 and this cut-off could be also important in the prognostic and therapeutic evaluation of the patient (8).

Microarray $\operatorname{IgE}$ assay inarguably represents an advancement in allergy diagnosis, as a third-level approach in poly-sensitized subjects, when the traditional diagnosis may be problematic. The use of the microarray leads to an improvement in the accuracy of diagnosis and appropriateness of treatment in a variable percentage ranging from 25 to $50 \%$ of patients, on the basis of the level of accuracy used for the molecular diagnosis (12).

Finally, when too many single recombinant allergens are required to define an accurate sensitization profile ISAC is preferable in terms of costs and efficiency, and particularly when 10 or more single recombinant allergens are required for diagnosis, microarray is economically superior (12).

\section{References}

1. Melioli G, Bonifazi F, Bonini S et al. Italian Board for ISAC (IBI). The ImmunoCAP ISAC molecular allergology approach in adult multi-sensitized Italian patients with respiratory symptoms. Clin Biochem. 2011;44(12):1005-11.

2. Valenta R, Lidholm J, Niederberger V, Hayek B, Kraft D, Grönlund $\mathrm{H}$. The recombinant allergen-based concept of component-resolved diagnostics and immunotherapy (CRD and CRIT). Clin Exp Allergy. 1999;29:896-904.

3. Sastre J. Molecular diagnosis in allergy. Clin Exp Allergy. 2010;40:1442-60.

4. Harwanegg C, Hiller R. Protein microarrays in diagnosing IgE-mediated diseases: spotting allergy at the molecular level. Expert Rev Mol Diagn. 2004;4(4):539-48.

5. www.thermofisher.com

6. Melioli G, Canonica GW. Molecular allergy diagnosis: we need to become more knowledgeable. Ann Allergy Asthma Immunol. 2012;108(6):387.

7. Asero R, Villalta D. Profilin may be a primary airbone sensitizer: a case report. J Invest Allergol Clin Immunol. 2013;23(2):134-5. 
8. Rossi RE, Melioli G, Monasterolo G et al. Sensitization profiles in polysensitized patients from a restricted geographical area: further lessons from multiplexed component resolved diagnosis. Eur Ann Allergy Clin Immunol. 2011;43(6):171-5.

9. Melioli G, Marcomini L, Agazzi A et al. The IgE repertoire in children and adolescents resolved at component level: a cross-sectional study. Pediatr Allergy Immunol. 2012;23(5):433-40.

10. Melioli G, Compalati E, Bonini S, Canonica GW. The added value of allergen microarray technique to the management of poly-sensitized allergic patients. Curr Opin Allergy Clin Immunol. 2012;12(4):434-9.
11. Bonini M, Marcomini L, Gramiccioni C et al. Microarray evaluation of specific IgE to allergen components in elite athletes. Allergy. 2012;67(12):1557-64.

12. Passalacqua G, Melioli G, Bonifazi $F$ et al and the Italian ISAC Study Group. The additional values of microarray allergen assay in the management of polysensitized patients with respiratory allergy. Allergy. 2013 in press.

13. Asero R. Component-resolved diagnosis-assisted prescription of allergen-specific immunotherapy: a practical guide. Eur Ann Allergy Clin Immunol. 2012; 44(5):183-7. 